\title{
Más allá de pajaritos y abejitas: sexualidad en el adolescente mexicano
}

\author{
Claudia Alejandra Cervantes Lara, Alicia Álvarez Aguirre \\ y María Mercedes Moreno Gónzalez
}

\begin{abstract}
Resumen
La adolescencia es una etapa de transición donde surgen cambios en cada una de las esferas que integran a una persona. Esto, aunado al crecimiento social acelerado, confronta a los adolescentes con nuevos desafíos respecto a su sexualidad. La falta de educación sexual integral desde la infancia y el tema de sexualidad tratado como un tabú permiten que se presenten consecuencias como el embarazo adolescente y enfermedades de transmisión sexual. Por lo tanto, se aborda dicha problemática, así como algunas estrategias que promueven el ejercicio de una sexualidad libre, informada y responsable.
\end{abstract}

Palabras clave: adolescencia, sexualidad, conducta sexual de riesgo, derechos sexuales, salud sexual.

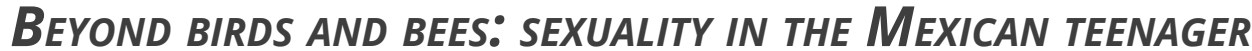

\begin{abstract}
Adolescence is a transition stage where changes arise in each of the spheres that make up a person. This, coupled with accelerated social growth, confronts adolescents with new challenges regarding their sexuality. The lack of comprehensive sexual education since childhood and the issue of sexuality treated as a taboo allow consequences such as teenage pregnancy and sexually transmitted diseases. Therefore, this problem is addressed, as well as some strategies that promote the exercise of free, informed and responsible sexuality.
\end{abstract}

Keywords: adolescence, sexuality, risky sexual behavior, sexual rights, sexual health. 
"Más allá de pajaritos y abejitas: sexualidad en el adolescente mexicano"

\section{Claudia Alejandra Cervantes Lara}

claudiacervantes43548@gmail.com https://orcid.org/0000-0003-0157-5423

Maestra en Ciencias de Enfermería, Universidad de Guanajuato, Campus Celaya - Salvatierra, División de Ciencias de la Salud e Ingenierías, Departamento de Enfermería y Obstetricia. Estudiante del Doctorado en Ciencias de Enfermería, Universidad de Guanajuato, Campus Celaya - Salvatierra, División de Ciencias de la Salud e Ingenierías, Departamento de Enfermería y Obstetricia.

\section{Alicia Álvarez Aguirre}

alicia.alvarez.aguirre@gmail.com https://orcid.org/0000-0001-5538-7634

Doctorado en Ciencias de Enfermería por la Universidad Autónoma de Nuevo León, Maestra en Ciencias de Enfermería por la Universidad Autónoma de Querétaro y Profesor de Tiempo Completo con adscripción al Departamento de Enfermería Clínica, División de Ciencias de la Salud e Ingenierías. Su trabajo incluye publicaciones en revistas indizadas y arbitradas, ponencias en congresos nacionales e internacionales, desarrollo de proyectos de investigación con financiamiento y evaluación de revistas mexicanas e internacionales. Ha dirigido tesis de pregrado y posgrado.

\section{María Mercedes Moreno Gónzalez}

ma.moreno@ugto.mx

http://orcid.org/0000-0002-0299-2299

Doctora en Ciencias de Enfermería, Universidad de Guanajuato, Campus Celaya - Salvatierra, División de Ciencias de la Salud e Ingenierías, Departamento de Enfermería y Obstetricia. Profesora de Tiempo Completo, Asociado B. Reconocimiento Perfil deseable PRODEP. (2017 - 2020) Certificado de Calidad como Enfermera Docente. Consejo Mexicano de Certificación de Enfermería A.C. Julio de 2018 - Julio de 2023). Coordinadora de práctica de Primer Nivel de Atención 
Imagen 1. Es importante el acercamiento a los jóvenes con el fin de conocer sus necesidades y expectativas, para con ello diseñar intervenciones en sexualidad que puedan resolver problemáticas actuales.

Cortesía de Cervantes-Lara.
Los adolescentes representan un importante porcentaje de la población mundial, situación también presente en México. Por ello, resulta importante conocer los cambios que ocurren durante esa etapa para entender las problemáticas de salud, culturales y psicológicas a las que se pueden enfrentar.

La Organización Mundial de la Salud (oms) sitúa a los adolescentes entre los 10 y 19 años de edad (oms, 2017a). Alrededor de 1,200 millones de personas en el mundo se encuentran en este rubro, lo que representa la sexta parte de la población total (oms, 2017b). Las estadísticas señalan que sólo en nuestro país existen más de 22.4 millones de adolescentes (Secretaría de Salud México, 2015).

Las definiciones de adolescencia se dan en distintos planos: sociológicos, psicológicos, fisiológicos, legislativos, económicos, tradicionales, cognitivos, cronológicos, entre otros (Sebald cit. en Borrás Santisteban, 2014); sin embargo, esta etapa está caracterizada por ser un período donde surgen diversos cambios que llevan a la persona a dejar la niñez para convertirse en adulto. Éstos ocurren en los aspectos físico, psicoemocional y social, lo que marca el comienzo de una búsqueda de identidad y de sentido de vida (UNICEF, 2017).

Es por ello que el término adolescencia suele ir acompañado de una visión negativa por parte de los adultos, ya que se trata de una etapa complicada pues, para algunos, todos estos cambios son difíciles de manejar, lo que se puede traducir en inestabilidad, estrés, búsqueda de independencia y necesidad de formar una identidad propia.

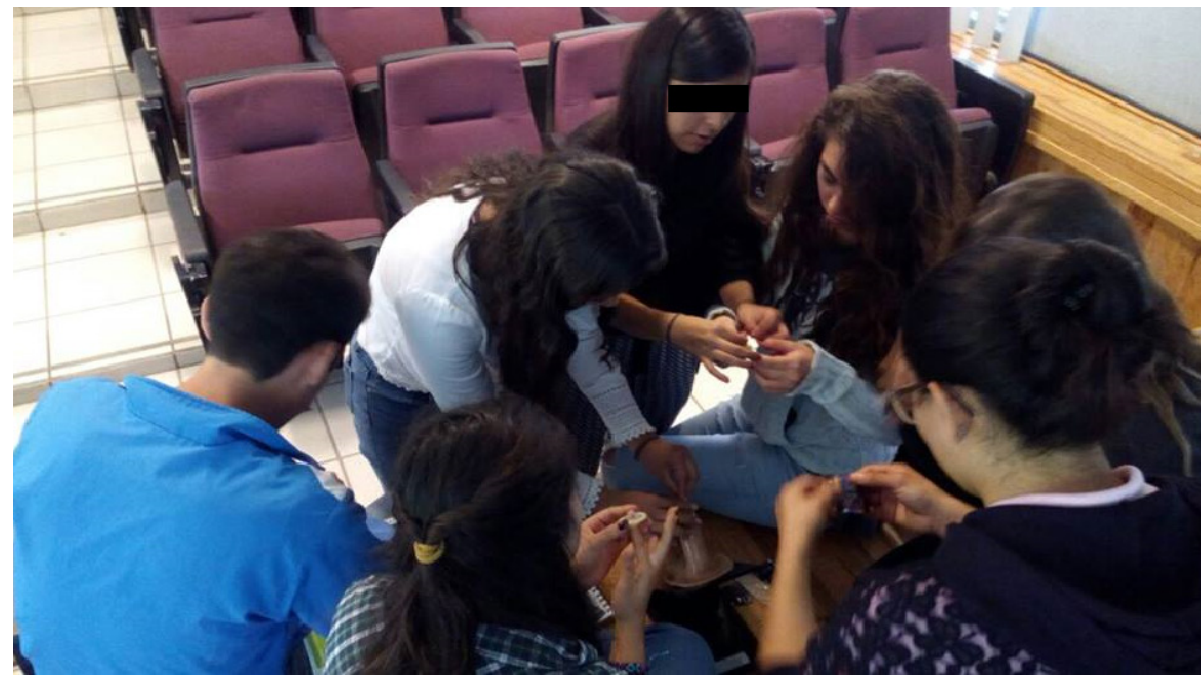

Aún más, durante la adolescencia, suele iniciarse la vida sexual, lo cual añadido a falta en el control de impulsos y labilidad emocional, pues el interés sexual aumenta al surgir la curiosidad por experimentar sensaciones nuevas (Romo 
Imagen 2. La inclusión del hombre en temas de uso de métodos anticonceptivos y de barrera, el embarazo, la interrupción legal del embarazo, etcétera, es de suma importancia para garantizar el ejercicio pleno de sus derechos sexuales y reproductivos. Cortesía de Cervantes-Lara.
Rodríguez, 2013) podría inclinar a los adolescentes hacia conductas de riesgo, en especial, vinculadas a las prácticas sexuales.

En esta etapa, comienza la lejanía con los padres; en cambio, los amigos se convierten en sus principales figuras (Gaete, 2015), y son ellos quienes toman parte importante en decisiones como la vestimenta, la elección de parejas sentimentales y el inicio o retraso de la actividad sexual. En ese sentido, los estudiantes que poseen amigos que practican conductas sexuales riesgosas (cSR) podrían tener una mayor probabilidad de también hacerlo (Forcada Mier et al., 2013).

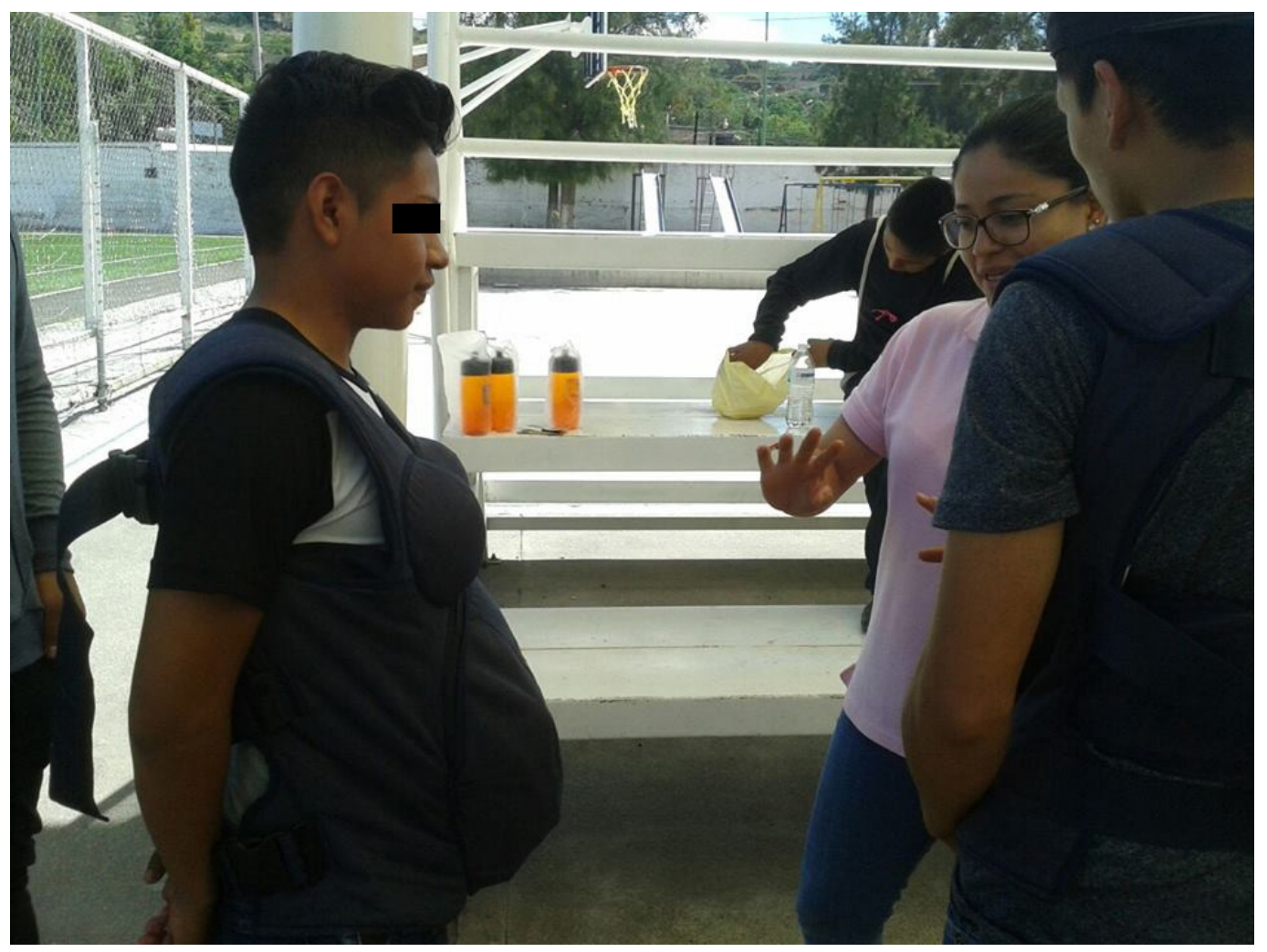

Entre las CSR encontramos, por ejemplo, el uso nulo o la falta constante de métodos de barrera como el condón y anticonceptivos, mantener relaciones sexuales bajo el uso de sustancias nocivas, tener actividad sexual con un alto número de parejas (Forcada Mier et al.,2013; Negeri, 2014) o con trabajadores sexuales (Golin et al., 2012), así como el inicio temprano de vida sexual. Dichas prácticas incrementan la posibilidad de contraer alguna enfermedad venérea o de un embarazo no deseado. Cabe destacar que estas conductas no sólo afectan la salud física del adolescente, sino que también lo hacen a nivel educativo, familiar, económico y social, tanto en el presente como en el futuro.

La existencia de estas conductas en México se vuelve clara al observar que las principales causas de muerte en adolescentes son complicaciones en el 
Imagen 3. La promoción de una educación sexual integral permite reconocer la importancia de una sexualidad saludable en la que se incluya el respeto a la diversidad, las prácticas sexuales, las infecciones de transmisión sexual, las alternativas de placer, el amor, el género, la capacidad reproductiva y los servicios a los que puede acercarse el adolescente para recibir atención y orientación acorde con sus necesidades.

Cortesía de Cervantes-Lara. embarazo, el suicidio, la violencia y los accidentes (oms, 2017b). En efecto, América Latina ocupa los primeros lugares con mayor tasa de embarazos adolescentes (Secretaría de Salud México, 2015). A su vez, la oms ha advertido que cada año se registran 44 nacimientos por cada 1000 chicas de entre 15 a 19 años (oms, 2017a), siendo este grupo el que mayor porcentaje de embarazos no planeados presenta: 73.9\% (INEGI, 2016).

El embarazo adolescente es un problema de salud pública, porque expone la salud física de la mujer y de su hijo. Sin embargo, esto no siempre es apreciado entre los adolescentes masculinos, quizá producto de los estereotipos sociales donde la culpa y responsabilidad del acto sexual recae, sobre todo, en la mujer.

Por otra parte, desde su despenalización, en abril del 2007, hasta abril del 2018 se han llevado a cabo en la Ciudad de México 208,353 servicios de aborto. No obstante, la interrupción del embarazo no es legal en todo el país, por lo que todavía se realizan abortos clandestinos, exponiendo a las mujeres a un alto riesgo de contraer infecciones, a perder su capacidad reproductiva o incluso la vida por una mala praxis (Grupo de Información en Reproducción Elegida, 2018).

De acuerdo con la Encuesta Nacional de la Dinámica Demográfica 2014, en nuestro país, la participación del hombre en el uso de los métodos anticonceptivos disminuyó de $23 \%$ a 19.8\%, cifra que puede estar justificada por la notable falta de inclusión en programas de salud (INEGI, 2016), y por los estereotipos, roles de género y normas sociales que limitan sus acciones. Además, la modernización de más métodos anticonceptivos exclusivos para mujeres podría segregar los procedimientos que involucran la colaboración, ya sea directa o indirecta, del hombre, como el uso del condón, la vasectomía, el retiro (coito interrumpido) y el ritmo o método de calendario (Rojas, 2014).

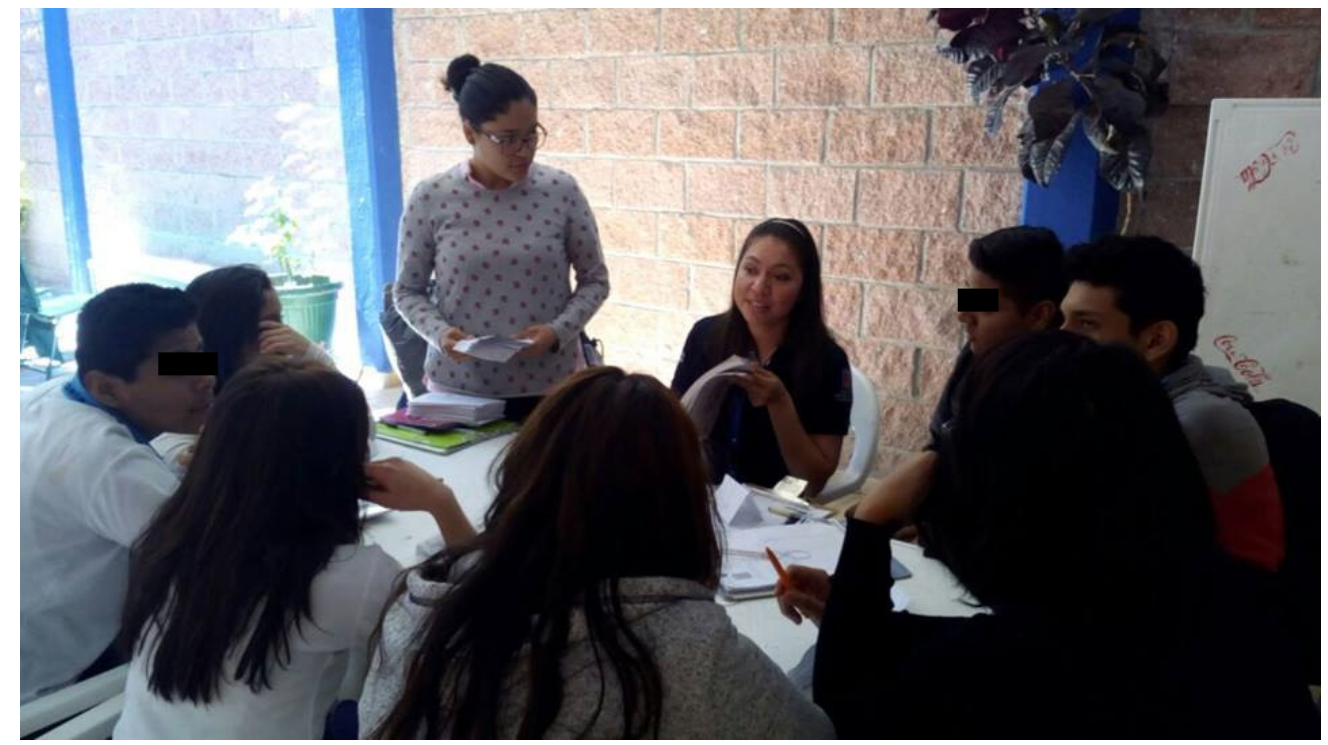


"Más allá de pajaritos y abejitas: sexualidad en el adolescente mexicano"

Una de las consecuencias de las cSR es la transmisión y adquisición del virus de inmunodeficiencia humana $(\mathrm{VIH})$, posicionado como la segunda causa de muerte en adolescentes (oms, 2016). Esto ha llevado a México a ser catalogado como un país en estado de epidemia con VIH (CENSIDA, 2015). De igual manera, datos de la oms revelan que más de un millón de personas cada día contraen una infección de transmisión sexual (ETS) y que existen 290 millones de mujeres infectadas en el área genital, oral o anal con el virus del papiloma humano (VPH) (OMS, 2016).

Las conductas sexuales riesgosas que suceden en la adolescencia terminan por repercutir en la vida adulta. De acuerdo con la oms, muchos de los problemas de salud en edades mayores comenzaron desde la juventud, producto del consumo de alcohol, infecciones de transmisión sexual, falta de ejercicio y una carente o inadecuada alimentación (oms, 2017b).

De acuerdo con las cifras nacionales de la Encuesta Nacional sobre Disponibilidad y Uso de Tecnologías de la Información en los Hogares (ENDUTIH), cerca de 62.4 millones de personas a partir de los seis años son usuarias de los servicios que ofrece internet (INEGI, 2014); lo cual, en conjunto con el uso de las redes sociales, podría facilitar conductas de riesgo.

A través de internet, los niños y adolescentes pueden estar en contacto con información errónea o no apta para su edad, acoso cibernético y visualización de contenido explícito. Por tanto, es de suma importancia realizar intervenciones con énfasis en el control del uso de medios electrónicos por parte de los padres o tutores.

Sobre este tema, Rice revela, a partir de su estudio Cell Phone Internet Access, Online Sexual Solicitation, Partner Seeking, and Sexual Risk Behavior Among Adolescents, que los estudiantes con acceso a internet tenían mayor probabilidad de ser sexualmente activos y de mantener relaciones sexuales con una persona con la que se pusieron en contacto en internet (2015). Lo anterior suele asociarse al empleo de aplicaciones diseñadas para encuentros sexuales, falta de control parental, alta exposición a medios electrónicos, presión social y búsqueda de nuevas experiencias por parte de los adolescentes.

No obstante, el acceso a toda esa información, por el contrario, podría favorecer que los adolescentes tengan mayor y mejor conocimiento sobre sexualidad, y puedan realizar decisiones informadas al respecto. Esto nos lleva a reflexionar, entonces, si el uso de internet y medios electrónicos es un factor protector o de riesgo para los adolescentes.

Por otra parte, al iniciar la vida sexual, también resulta importante estar consciente sobre la posibilidad de un embarazo y de lo que conlleva, pues si bien esta decisión forma parte de los derechos sexuales y reproductivos, el contar con el nivel máximo de salud también lo es. En consecuencia, brindar información y facilitar el acceso a los diversos métodos de barrera y anticoncepción tiene un 
"Más allá de pajaritos y abejitas: sexualidad en el adolescente mexicano"

papel crucial para una elección libre pero informada. En ese sentido, los profesores y personal de salud pueden fomentar en el adolescente la construcción de un proyecto de vida, motivarlo a visualizar claramente sus metas y los impactos de cada acción.

Acerca del embarazo, se considera necesario un acompañamiento continuo y personalizado, que considere los aspectos culturales de adolescente, como su religión, estado físico, emocional y económico. Para ello se sugiere recibir apoyo psicológico, así como asesoría gratuita para el control prenatal o sobre las clínicas de aborto regularizadas.

En relación con los hombres, quienes cuentan con menor número de métodos anticonceptivos, se deberá concientizar sobre su derecho de decidir si quiere tener hijos, cuántos y el espaciamiento entre ellos. Esto implica, por supuesto, el compromiso de conocer qué opciones hay para el control de paternidad, la desmitificación de los efectos adversos de la vasectomía y acabar con los estereotipos sociales que impiden ejercer una sexualidad responsable, libre e informada.

\section{Conclusiones}

La adolescencia es sin duda una de las principales etapas de cambio dentro de la vida humana, lo que puede traer consigo confusión, problemáticas sociales, familiares y emocionales; ya que la mayoría de las veces el joven carece de comprensión por parte de quienes lo rodean. Por ello, se deben implementar estrategias desde el ámbito familiar, en donde se dé información a temprana edad sobre los riesgos de practicar conductas de riesgo que pueden perjudicar la salud física y mental. Asimismo, se deben considerar los cambios sociales a los que se enfrenta para aumentar la autoeficacia y la autoestima en el adolescente, con fin de que sea capaz de afrontar cualquier situación.

De este modo, toda perspectiva orientada al tema debe incidir en que los adolescentes tomen decisiones con el conocimiento de sus derechos. La cuestión radica en promover el ejercicio de estos derechos estando consciente de sus posibles consecuencias.

El conocimiento de la sexualidad y el ejercicio de la misma han sido vistos desde una perspectiva negativa, lo que orilla a los adolescentes a iniciar vida sexual de manera secreta, disminuyendo la posibilidad de adquirir métodos de protección. Este hecho también repercute en la falta de autoconocimiento, producto de una carente autoexploración; ejemplo de ello es que enfermedades prevenibles como cáncer de mama o cervicouterino van en aumento porque la mayoría población no sabe si una protuberancia en su cuerpo es normal o cuánto tiempo tiene de haber aparecido. 
La sexualidad es un tema muy amplio que requiere ser tratado a edades tempranas para que cuando el individuo llegue a la adolescencia cuente con las herramientas necesarias para lograr asertividad sexual. Además, es necesario conocer el cuerpo a través de la autoexploración y de la masturbación, decidir libremente sobre la orientación sexual, conocer la diversidad de prácticas para obtener placer, siempre y cuando representen bajo riesgo de contagio y embarazo, y estar al tanto de los lugares donde pueden acercarse para recibir atención de manera gratuita.

La adolescencia, a pesar de los retos y desafíos, es el momento en el que se puede disfrutar de la compañía de amigos, experimentar la emoción de la primera pareja, escoger los estudios a realizar, viajar, desarrollarse académicamente y disfrutar plenamente de actividades recreativas. Con consciencia sobre la sexualidad, los métodos anticonceptivos y las conductas de riesgo, además de un ambiente familiar y social en el que se promueva el conocimiento sobre los derechos sexuales y sus implicaciones, en conjunto con autoconocimiento y autoexploración, contaremos con adolescentes que podrán enfrentarse a los nuevos desafíos. ¿Será esto posible? ¿Hay que considerar otras alternativas? Una cosa es segura, debemos tratar la sexualidad más allá de pajaritos y abejitas.

\section{Información de interés}

- \#YoSímeCuido

- Asociación Mexicana para la Salud Sexual

- ¿Cómo le hago?

- Servicio de orientación en salud UNAM

- Programa de Prevención de Embarazo en Adolescentes UNAM

\section{Referencias}

* Borrás S. T. (2014). Correo Científico Médico, 18(1), 05-07. Dol: http://scielo.sld.cul scielo.php?pid=S1560-43812014000100002\&script=sci arttext\&tlng=pt.

* Censida. (2015). Informe Nacional de Avances en la Respuesta al VIH y el SIDA. Recuperado de: https://www.gob.mx/censida/documentos/informe-nacional-deavances-en-la-respuesta-al-vih-y-el-sida-mexico-2015.

- conapo. (2014). Salud Sexualy Reproductiva Estado de México. Recuperado de: http:// www.gob.mx/conapo.

* Forcada M. P., Pacheco A. S., Pahua M. E., Palacios R. P., Todd F. E., \& Pulido R. A. (2013). Conducta sexual de riesgo en estudiantes universitarios: factores de riesgo y protección. Revista Intercontinental de Psicologia y Educación, 15(1), 23-46. Recuperado de: https://www.redalyc.org/articulo.oa?id=80225697003. 
"Más allá de pajaritos y abejitas: sexualidad en el adolescente mexicano" Claudia A. Cervantes Lara, María M. Moreno Gónzalez y Alicia Álvarez Aguirre

Vol. 21, Núm. 2, marzo-abril 2020 Revista Digital Universitaria

Gaete, V. (2015). Desarrollo psicosocial del adolescente. Revista Chilena de Pediatría, 86(6), 436-443. Recuperado de: https://scielo.conicyt.cl/pdf/rcp/v86n6/art10.pdf.

* Golin, C. E., Earp, J. A., Grodensky, C. A., Patel, S. N., Suchindran, C., Parikh \& MGroves, J. (2012). Longitudinal Effects of SafeTalk, a Motivational InterviewingBased Program to Improve Safer Sex Practices Among People Living with HIV/AIDS. Alds and behavior, 16(5), 1182-1191. Dol: https://doi.org/10.1007/s10461-011-00259.

* Grupo de Información en Reproducción Elegida (GIRE) (2018). Mitos y preguntas sobreelaborto. Recuperado de:https://gire.org.mx/wp-content/uploads/2019/08/ mitos y preguntas sobre el aborto.pdf.

* Instituto Nacional de Estadística y Geografía (INEGI). (2014). Encuesta Nacional sobre Disponibilidad y Uso de Tecnologías de la Información en los Hogares (ENDUTIH). Recuperado de: https://www.gob.mx/cms/uploads/attachment/ file/63383/Resultados ENDUTIH-Prensa INEGI rev 11032015.pdf.

* Instituto Nacional de Estadística y Geografía (INEGI). (2016). Encuesta Nacional de la Dinámica Demográfica 2014 (ENADID). Recuperado de: https://www.inegi.org.mx/ programas/enadid/2014l.

* Negeri, E. L. (2014). Assessment of risky sexual behaviors and risk perception among youths in Western Ethiopia: The influences of family and peers: A comparative cross-sectional study. bmc Public Health, 14(1). Dol: https://doi. org/10.1186/1471-2458-14-301.

* Organización mundial de la salud (oms). (2016). Infecciones de transmisión sexual. Recuperado de: http://www.who.int/mediacentre/factsheets/fs110/es/.

* Organización mundial de la salud (oms). (2017a). Adolescentes: riesgos para la salud y soluciones. Recuperado de: http://www.who.int/mediacentre/factsheets/ fs345/es/.

* Organización mundial de la salud (oms). (2017b). Salud de los adolescentes. Recuperado de: http://www.who.int/topics/adolescent health/es/.

* Pulido Rull, M. A., Ávalos Tinoco, C., Fernández Mondragón, J. C., García Flores, L., Hernandez Muñoz, L. \& Ruiz Campos, A. C. (2013). Conducta sexual de riesgo en tres universidades privadas de la Ciudad de México. Psicología y Salud, 23(1), 25-32. Recuperado de: https://pdfs.semanticscholar.org/4700/ cfb164fc2d630255d7be2004110d9617fc85.pdf.

* Rice, E., Winetrobe, H., Holloway, I. W., Montoya, J., Plant, A., \& Kordic, T. (2015). Cell Phone Internet Access, Online Sexual Solicitation, Partner Seeking, and Sexual Risk Behavior Among Adolescents. Archives of Sexual Behavior, 44(3), 755-763. Dol: https://doi.org/10.1007/s10508-014-0366-3.

- Rojas, O. (2014). Los hombres mexicanos y el uso de anticoncepción. Iztapalapa, Revista de Ciencias Sociales y Humanidades, (77), 77-95. Recuperado de: https:// www.redalyc.org/pdf/393/39348246004.pdf. 
"Más allá de pajaritos y abejitas: sexualidad en el adolescente mexicano" Claudia A. Cervantes Lara, María M. Moreno Gónzalez y Alicia Álvarez Aguirre

Vol. 21, Núm. 2, marzo-abril 2020 Revista Digital Universitaria

* Romo Rodríguez. (2013). Embarazo Adolescente [tesis para obtener el grado de médico, Universidad San Francisco de Quito]. Recuperado de: http://repositorio. usfa.edu.ec/bitstream/23000/2171/1/106912.pdf.

* Secretaría de Salud México. Modelo de Atención Integral en Salud Sexual y Reproductiva para adolescentes. Recuperado de: https://www.gob.mx/cms/ uploads/attachment/file/181050/web ModAtnIntSSRA.pdf.

* Secretaría de Salud México. (2015a). Estrategia Nacional para la Prevención del Embarazo en Adolescentes. Recuperado de: https://www.gob.mx/cms/uploads/ attachment/file/232826/ENAPEA 0215.pdf.

* unICEF (2017). Para Cada Adolescente una Oportunidad. Recuperado de: https:// www.unicef.org/argentina/media/1396/file/Posicionamiento\%20adolescentes. pdf.

World Health Organization (wHo). (2014). Health for the World's Adolescents. A second chance in the second decade. Recuperado de: www.who.int/adolescent/ second-decade.

* Wilson-Mitchell, K., Bennett, J., \& Stennett, R. (2014). Psychological health and life experiences of pregnant adolescent mothers in Jamaica. International Journal of Environmental Research and Public Health, 11(5), 4729-4744. Dol: https://doi. org/10.3390/ijerph110504729.

\section{Cómo citar este artículo}

- Cervantes Lara, Claudia Alejandra, Álvarez Aguirre, Alicia y Moreno Gónzalez, María Mercedes (2020). Más allá de pajaritos y abejitas: sexualidad en el adolescente mexicano. Revista Digital Universitaria (RDU). Vol. 21, núm. 2 marzo-abril. Dol: http:// doi.org/10.22201/codeic.16076079e.2020.v21n2.a7.

Recepción: 11/04/2018. Aprobación: 23/09/2019 